\title{
Civilisations
}

Revue internationale d'anthropologie et de sciences

humaines

59-1| 2010

Sexualités : apprentissage et performance

\section{Une initiation diffuse à la sexualité}

Le sabar des Wolof du Sénégal

\section{Audrey Dessertine}

\section{(2) OpenEdition}

12 Journals

Édition électronique

URL : http://journals.openedition.org/civilisations/2245

DOI : 10.4000/civilisations. 2245

ISSN : 2032-0442

Éditeur

Institut de sociologie de l'Université Libre de Bruxelles

\section{Édition imprimée}

Date de publication : 28 juin 2010

Pagination : 89-108

ISBN : 2-87263-029-5

ISSN : 0009-8140

\section{Référence électronique}

Audrey Dessertine, «Une initiation diffuse à la sexualité », Civilisations [En ligne], 59-1 | 2010, mis en ligne le 29 juin 2013, consulté le 30 avril 2019. URL : http://journals.openedition.org/civilisations/2245 ; DOI : 10.4000/civilisations.2245 


\title{
Une initiation diffuse à la sexualité Le sabar des Wolof du Sénégal
}

\author{
Audrey DESSERTINE
}

Résumé : À Dakar, la danse est présente à tous les âges de la vie de nombreuses femmes wolof, et cela dès la plus tendre enfance. Si toutes ne dansent pas dans les réunions féminines où se danse le sabar, beaucoup sont appelées à participer à ces moments de sociabilité où se donne à voir, à travers les mouvements dansés, une image saisissante de la sexualité. En effet, le sabar célèbre la sexualisation du corps tout en produisant une étonnante parodie des rapports entre les sexes qui passe non seulement par un jeu sur les conventions mais aussi par la mise en scène d'une forme grotesque de sexualité. Toutefois, s'il est un moment de jeu et de détente, le sabar n'en est pas moins dûment codé : il exige une maîtrise de techniques du corps que les fillettes s'exercent à reproduire en observant leurs aînées. Ainsi, l'article traite de la question de la fabrique sociale des corps féminins, de l'apprentissage d'une certaine hexis corporelle ainsi que des modalités singulières et souvent paradoxales de cet apprentissage, censé transformer une "enfant du lignage » en une femme confrontée à la question de l'union sexuelle.

Mots-clés : Sénégal, sabar, sociabilité féminine, apprentissage, sexualité, danse.

Abstract: In Dakar, dance is part of the life of most wolof women. All women do not dance regularly in feminine meetings, but most of them participate in moments of sociability where sabar dance is performed, displaying a surprising image of sexuality. Indeed, sabar performances celebrates the sexualisation of the body and simultaneously produces a parody of the relations between genders in playing with conventions and in staging a grotesque form of sexuality. However, if sabar performances are moments of play and entertainment, they also demand to master specific bodily techniques that young girls progressively learn in observing and imitating (young) women. Drawing on ethnographical fieldwork conducted in Dakar and in Paris, the article evokes the social making of feminine bodies, the learning of a bodily hexis as well as the specific and paradoxical modalities of this process of embodiment, which progressively transforms a "child of the lineage" into a woman confronted to the issue of sexuality.

Key words: Senegal, sabar, feminine sociability, learning, sexuality, dance. 


\section{Introduction}

L

es modes de socialisation de la sexualité et les codifications dont celle-ci fait l'objet dans les sociétés africaines ont été abordés par les anthropologues sous de multiples aspects : éducation précoce au comportement propre à son genre ${ }^{1}$, prescriptions et interdits sexuels (notamment Echard, Journet, Lallemand 1981 : 337-395), interventions sur les organes génitaux, mises en scène rituelles (en particulier dans le cadre de rites initiatiques), etc. Plus rares sont les études mettant au cœur de l'observation une technique corporelle ${ }^{2}$ telle que la danse, dans ses rapports directs avec la sexualité.

Performance non verbale qui condense de multiples dimensions esthétiques, sociales et symboliques, la danse met en lumière différentes stratifications de la société : statuts particuliers, rapports sociaux d'âge et de sexe (voir, sur ces questions dans la société wolof, Le Cour Grandmaison 1967 ; Le Carme 2000). Elle est l'expression de codes de convenance et, comme nous le verrons ici, de jeux sur les limites qui passent par le corps, un corps qui, loin d'être clos sur lui-même, est ce qui permet la relation au monde. Si la danse est un langage, comment penser en « texte » cet indicible traduit par corps (Legendre 1978) ? Toute danse suppose une chorégraphie, soit un protocole que les danseuses mettent en œuvre. Pour que ce corps soit écouté et saisi, il faut faire en sorte qu'il résonne en caractères lisibles. $\mathrm{Si}$ les mouvements dansés sont très codifiés et s'inscrivent dans un rapport de correspondance avec le code musical, le sabar est aussi une danse chargée de significations sociales, de représentations et de modes d'agir qui ne prennent tout leur sens qu'au regard des normes qui continuent à se transmettre dans la société. Danse sans cesse réinventée, le sabar est loin d'être une tradition figée. De nouveaux mouvements apparaissent quotidiennement, intrinsèquement liés aux créations musicales des griots percussionnistes ${ }^{3}$. À partir de l'analyse des gestes symboliques observés dans le sabar, quelle place cette danse occupet-elle parmi les différents procédés qui contribuent à construire socialement la sexualité ? C'est à cette question que ce texte apporte des éléments de réponse.

Terme polysémique, sabar désigne au Sénégal à la fois un ensemble de tambours, une danse et les diverses occurrences (réunions festives) au sein desquelles il est utilisé. Il tient

1. Parmi les nombreux ouvrages qui accordent une attention particulière au façonnement précoce du genre, on citera, pour la société wolof, les ouvrages de Collette Le Cour Grandmaison (1967, 1978).

2. M. Mauss est le premier anthropologue, en 1934, à témoigner de la valeur heuristique du concept des techniques du corps. Il établit un lien entre les techniques corporelles, la socialisation et la culture.

3. Pour prendre un exemple, aujourd'hui, de nombreux musiciens affirment que les rythmes sont joués plus rapidement qu'avant. Ce changement de tempo influence la danse : certains mouvements disparaissent et laissent place à d'autres, plus adaptés. Les danseuses doivent donc faire preuve d'une plus grande maîtrise et d'une importante dextérité pour parvenir à danser dans le temps. 
son origine de la société wolof ${ }^{4}$. Faisant partie intégrante de nombreuses cérémonies, le sabar permet d'interroger les codes sociaux intervenant directement dans la constitution de l'hexis corporelle des femmes wolof, tout comme il révèle la place de représentations et de ritualités étrangères à l'Islam dans une société très largement islamisée. Au Sénégal, comme dans les populations immigrées en France, il fait partie intégrante de nombreuses cérémonies familiales ${ }^{5}$ (pour célébrer un mariage ou un baptême), il peut aussi être organisé lors de réunions féminines ou de fêtes de rue. Quelle qu'en soit l'occurrence, toute séance de danse est indissociable des tambours, des rythmes et des devises tambourinées qui l'accompagnent de manière nécessaire.

Sept années de pratique régulière de la danse sabar à Paris ont favorisé mes observations et mes analyses en contexte migratoire. Mais c'est surtout à partir d'observations et de la pratique de la danse à Dakar au Sénégal dans les quartiers populaires de la Médina et de la Gueule Tapée, que je poursuis aujourd'hui mes travaux.

Dans cet article, je m'interroge particulièrement sur ce qui donne au sabar sa tonalité si particulière dans une société qui valorise par ailleurs si fortement les notions de respect, de pudeur (kersa), et de patience (mugn), et ce autant dans l'éducation d'une jeune fille que dans la reconnaissance des qualités qui font la réputation d'une femme. Ici, on joue de toute la gamme des techniques de séduction féminine : tissus, fards, jeux de regards, gestuelle. Des corps exacerbés dans leur féminité et une représentation « crue » de la sexualité, tel est le donné à voir le plus saillant du sabar wolof, dansé par les femmes. En produisant d'étonnantes mises en scène de la sexualité (imitations du coït, exhibition de sexes d'homme en bois ou en plastique) ${ }^{6}$, on essaiera de voir comment le sabar questionne et célèbre la sexualisation du corps et de quelle forme d'éducation il est porteur.

\section{Hexis corporelle et sociabilités féminines}

Dès la petite enfance, l'éducation est, dans la société wolof, pensée différemment selon le sexe, et prépare progressivement les enfants à actualiser, respectivement, une certaine image de la virilité et de la féminité ${ }^{7}$. Il existe des qualités inhérentes à la féminité à partir desquelles s'inscrivent les rôles sociaux féminins et les valeurs qui orientent les comportements et les

4. Sur la société wolof, l'ouvrage de référence reste celui d'Abdoulaye Diop (1981). Le domaine traditionnel des wolof s'étend du nord au sud, depuis le delta du Sénégal jusqu'à la latitude de jurbel, limite septentrionale approximative du pays sereer, et d'ouest en est, de la côte atlantique au désert du ferlo. Les régions qu'il englobe ont constitué, dans le passé précolonial, les royaumes du waalo, du jolof, du kajoor et du baol. Aujourd'hui encore, les wolof occupent principalement cet espace géographique, et voisinent au nord avec les maures et les tukuloor, au sud avec les serer et à l'est avec les pël; des minorités issues de ces ethnies vivent au milieu d'eux, souvent depuis des siècles. Aujourd'hui, les wolof constituent le groupe le plus important du Sénégal, du point de vue démographique (en 1988, ils représentaient 43,7\% de la population sénégalaise). Leur importance culturelle est aussi une réalité, l'expansion générale de leur langue en est un signe. Il s'avère que plus de $80 \%$ des sénégalais parlent wolof, comme première ou deuxième langue.

5. Les implications de ces cérémonies quant à la réputation d'une femme ont été très finement analysées par Fatou Sow (1976) dans son étude sur la teranga.

6. De telles scènes avaient frappé l'attention de Collette Le Cour Grandmaison (1978), lorsqu'elle travaillait en milieu dakarois dans les années 1960.

7. Jacqueline Rabain note que le sevrage est une opération rituelle qui inaugure une série de transformations dont l'accès à une identité sexuée spécifiée (1979: 44). 
choix. Les principes qui régissent l'éducation des enfants fixent les modalités des relations qu'ils entretiennent avec les membres de leur sexe ou du sexe opposé. L'éducation donnée aux petites filles comprend très vite l'accomplissement de certaines tâches domestiques et la surveillance des petits frères et sœurs. Les jeunes filles apprennent à être réservées, respectueuses et patientes envers les hommes et les aînés des deux sexes. Jacqueline Rabain, dans son ouvrage sur l'enfance en pays wolof, note que vers quatre ans déjà, on attend de l'enfant qu'il se classe dans une génération parmi les enfants de même âge. Il apprend alors à garder ses distances à l'égard des aînés et des adultes en général. C'est dans la compagnie des enfants de même âge, dans l'écoute discrète des aînés et des adultes, que se développera le sens de la séduction et que s'inscrira en même temps la nécessité de la présence et des contacts tactiles (Rabain 1979 : 20). La relation qui lie une jeune fille à un autre membre du même sexe et de même âge est donc la seule relation qui est vécue, au sein de cette société hiérarchisée, sous le signe de l'égalité. C'est sans doute une des seules relations où l'improvisation, la fantaisie et la liberté peuvent s'exprimer (expression qui prend une grande importance dans la vie sociale féminine). Ainsi, les jeunes filles d'un même groupe d'âge ${ }^{8}$ entretiennent des relations vécues sur un mode affectif et leur attachement s'actualise par de nombreux contacts corporels. Les corps en contact forment un espace de co-présence, où les attouchements, les regards, les positions corporelles relâchées, concourent à créer une intimité partagée. Ce mode de contact corporel est aussi un moment d'apprentissage de la beauté et de la séduction. Il s'agit de rendre belle son amie, sa sœur, en façonnant son apparence. Une fois adultes, les mariées et les jeunes mères entrent, lors des baptêmes, dans un système d'échanges de cadeaux, qui propage des modèles de beauté importés, diffusés par les médias comme les crèmes de défrisage à froid, le xeesal et autres produits éclaircissant la peau, le brillant à lèvres, le fard à paupières. Ces produits transforment les visages à la manière d'un véritable marquage symbolique. Ils peuvent s'associer aussi aux canons coutumiers de la coquetterie qui valorisent les tresses, la façon de nouer son mouchoir de tête, les bijoux en or. Ces signes renvoient à des modes de consommation révélant des processus d'identification différenciés selon les groupes d'âge. Les jeunes filles choisiront de s'habiller à l'occidentale ou de revêtir une tenue unique, dessinée par leur soin chez le tailleur ${ }^{9}$ tandis que les femmes d'âge mûr opteront pour un élégant boubou taillé dans un bazin riche.

Durant les sorties en ville, les jeunes filles actualisent de nouveau leur attachement en se tenant la main. Ce mode de contact, en public, est également observable entre jeunes garçons. En effet, le mode tactile d'expression de l'attachement n'est permis qu'entre jeunes gens du même sexe. L'engagement mutuel des corps n'est pas possible entre homme et

8. Les wolof connaissaient un système de classes d'âge, organisant selon un principe linéaire garçons et filles par échelon d'âge. Il ne reste plus aujourd'hui que des débris de ce système et un terme de référence dont l'utilisation est devenue assez vague. C'est le terme « maas» par lequel on désigne à partir de 4-5 ans le compagnon de même âge d'un enfant ou encore les compagnes de la jeune épousée. Des activités ludiques des maas ont subsisté, telles des fêtes qu'organisent de temps en temps, à partir de cotisations, garçons et filles d'un même quartier. Ces fêtes sont chantées et dansées (Rabain 1979: 218).

9. L'hiver 2008-2009, un engouement pour la mode « Bollywood» poussa les jeunes femmes dakaroises à rivaliser d'inventivité pour concevoir des tenues inspirées du sari et de la kurta indienne, confectionnées dans des voiles satinés aux couleurs vives et agrémentés de perlages brillants. Les danseuses tournoyaient dans la nuit, telles des papillons scintillants attirés par la lumière. 
femme dans la sphère publique. Par contre, les relations qui lient une jeune fille à une autre femme plus âgée sont placées sous le signe de l'obéissance et du respect, tout comme les relations entre mari et femme, vécues en public sous le signe de la pudeur et de la réserve. Toutefois, pour les jeunes filles, certaines relations d'amitié et de confidence avec des jeunes femmes un peu plus âgées ayant déjà de l'expérience sont parfois le moyen de prendre connaissance de la sexualité et des relations intimes entre hommes et femmes. Aujourd'hui en milieu urbain, j'ai observé que les jeunes gens des deux sexes partagent des moments privilégiés, usent d'une liberté de parole pour communiquer (notamment sur les sujets liés à la sexualité) et ont des contacts physiques, ceci n'étant possible qu'en l'absence d'aînés : on se chamaille, on se cherche et l'on se séduit. Les médias jouent également un rôle non négligeable aujourd'hui dans l'éducation sexuelle des jeunes à Dakar, qu'il s'agisse de la TV, d'internet ou des DVD. Les corps et notamment la sexualité auparavant tenus secrets (ou du moins ne faisant pas l'objet de ce type de monstration) s'imposent par l'image et laissent voir une nouvelle perception des relations sexuelles. Les films pornographiques connaissent une diffusion grandissante, sans réel contrôle et avec une relative facilité d'accès. Les jeunes se les procurent sans difficulté sur les marchés et par des réseaux d'amis. Les jeunes de même génération et de même milieu social se retrouvent très souvent pour partager des moments de loisirs. Dans ce cadre, certains organisent des séances de visionnage en groupe et parfois aussi en compagnie des petites amies ${ }^{10}$.

L'entre soi féminin se cultive tout au long de la vie d'une femme. En milieu wolof, il s'actualise notamment lors des nombreuses réunions d'associations d'âge ou d'affinités qui fonctionnent sur le mode de la tontine. Très développées au Sénégal comme dans toute l'Afrique de l'Ouest, les tontines féminines correspondent à une séparation marquée entre les sphères d'activité féminine et masculine. Elles sont imbriquées dans les échanges occasionnés par les cérémonies familiales ${ }^{11}$. Ces réunions, dans la continuité des groupements par classe d'âge, pratiqués dès l'enfance et au plus près de la vie sociale courante, présentent les traits marquants de groupes primaires : nombre restreint, présence directe, relations intenses, forte solidarité et rôle dans la vie morale. Ce sont des ateliers de reproduction et d'invention sociale qui doublent potentiellement tout le champ social d'un individu. Ainsi, Francesca Lulli a pu mettre en évidence les liens étroits qui unissent pouvoir économique, sociabilité et séduction (Lulli 2003). La tontine permet de rendre actif ce pouvoir à travers la mise à disposition d'une somme qui est d'une importance cruciale pour l'affirmation de la femme dans la société, tant du point de vue économique que du point de vue relationnel. La danse est très souvent présente au sein de ces rencontres et elle y joue un rôle important. Elle permet de célébrer et de faire l'expérience d'un rapport au corps spécifique durant ces moments d'intimité. Ces réunions, parmi leurs diverses caractéristiques, ont la prérogative de créer un espace-temps qui permet de ratifier des formes de solidarité, de contribuer à former un tissu social grâce au partage d'intérêts et à des modalités d'entraide. Les relations

10. Il serait pertinent de faire une analyse fine de l'impact de ces images, notamment sur les représentations que se font les jeunes des rapports sexuels et plus généralement, des relations entre les sexes.

11. La tontine est une association rotative d'épargne et de crédit. Le terme courant pour désigner une tontine en wolof est natt (une somme égale versée par chacun) ou tegg, littéralement « poser une mise ». Sur ce sujet, voir l'article de Jeanne Semin (2007). Pour une description des transactions et des enjeux économiques des cérémonies familiales, voir l'article d'Ismaël Moya (2004). 
entre femmes sénégalaises, dans ces moments privilégiés, ont pour normes principales l'absence de contraintes et la démonstration de la dimension affective. Les femmes se retrouvent entre elles pour vivre un moment fort d'amitié, de joie et de plaisir partagé. Les tontines sont des espaces de parole où les conversations permettent d'aborder des sujets délicats (l'espacement des naissances, la polygynie, le divorce, la sexualité, les sentiments amoureux, les relations avec les maris, les amants, les conflits entre co-épouses) où la vie des femmes est engagée. Les langues se délient, on célèbre une beauté exaltée par les soins corporels et les parures, les corps jouent et dansent.

\section{Une scène singulière : la danse sabar au cœur des réunions féminines}

Si le sabar se pratique en diverses occasions et se déroule dans des espaces divers, je m'intéresse ici spécifiquement aux réunions féminines. C'est en effet dans ce contexte que les danseuses pratiquent les mouvements les plus osés. Commençons par un aperçu de deux scènes de sabar auxquelles j'ai assisté à Dakar, en février 2006.

La première se déroule à la tombée de la nuit (après la prière de 17h) dans une cour fermée du quartier de la Médina, au cœur d'un espace clos et privé. Devant la porte, des jeunes filles " filtrent» le passage. À l'intérieur, une cinquantaine de femmes sont assises en cercle, dans un espace délimité par des chaises. Elles sont très serrées et proches les unes des autres, certaines sont installées sur des pagnes étendus au sol, aux pieds de leurs amies, tenant dans leurs bras de jeunes enfants. Les musiciens ${ }^{12}$ sont sept : quatre jouent les tambours du sabar ${ }^{13}$ et trois sont des joueurs de tambours d'aisselle ${ }^{14}$. La majorité des femmes porte des tenues unies et identiques, constituées d'un pagne et d'une tunique. La moitié de l'assistance est revêtue d'une parure réalisée dans un bazin orange, tandis que l'autre moitié porte une tenue confectionnée avec le même tissu, mais de couleur jaune. Les femmes tiennent également des sifflets et des bonbons aux couleurs assorties. Deux équipes sont ainsi présentées et s'affrontent amicalement par la danse. Cette mise en scène n'illustre pas une réelle compétition, elle sert surtout à alimenter l'ambiance et à créer ainsi une atmosphère de fête. Dès qu'une jeune femme de l'équipe jaune danse, toutes les autres, vêtues de même, l'encouragent en sifflant et en jetant des bonbons en l'air ${ }^{15}$.

12. Jouer des tambours est une activité masculine par excellence. Les musiciens sont, la plupart du temps, les seuls hommes présents durant ces réunions féminines. Parfois les organisatrices payent un photographe ou un caméraman pour filmer la fête et proposer des portraits aux participantes.

13. La batterie de sabar est constituée de différents tambours qui connaissent un rôle spécifique dans la polyrythmie qui accompagne la danse :

- Le ndeer (le tambour qui dirige et marque les pas de la danseuse)

- Le mbeu mbeu et le tumguni (rôle d'accompagnement)

- Le lamb et le col (les deux tambours qui tiennent le rôle de basse)

14. Le tama est un tambour d'aisselle qui n'est pas spécifique à la société wolof contrairement aux tambours qui constituent la batterie de sabar; on le retrouve dans de très nombreuses régions d'Afrique et notamment dans toute l'A frique de l'Ouest. Il joue cependant un rôle essentiel dans chaque cérémonie où la danse sabar est présente. Le terme tamakat désigne les joueurs de tama.

15. Notons qu'il existe souvent des concours organisés durant ces rencontres entre femmes. La gagnante elle celle qui danse le mieux ou qui porte le plus joli petit pagne. Le premier prix est souvent un lot constitué de tissus et de produits de beauté, soit autant d'objets utilisés dans la mise en valeur de la beauté et du charme féminins. 
Les danseuses, après s'être entraînées aux différents rythmes du sabar ${ }^{16}$, reconnaissent celui qui clôture toute séance de danse : le lëmbël. Il existe mille et une façons de danser sur ce morceau également appelé « ventilateur». Ici, c'est le lëmbël «couché » qui est réalisé. Les femmes installent alors un pagne, un petit pagne (beeco) rose appelé filet, qu'elles étalent sur le sol face aux joueurs de tama. Une première s'allonge sur le ventre et effectue la danse du ventilateur au sol sur le pagne installé pour la danse. Elle roule les fesses et soulève le bassin en saccades violentes avec une grande précision et une impressionnante agilité. Elle répond en interaction directe avec le tama ndeer ${ }^{17}$ qui est très proche d'elle et ne quitte pas des yeux les mouvements des hanches et du bassin. Les danseuses se succèdent et certaines femmes relèvent leur pagne tout en s'installant au sol : les beeco, les strings et même les fesses sont à la vue de l'assemblée et des musiciens. Certaines s'amusent à remonter le petit pagne d'une exécutante, trop timide. Souvent, une autre femme arrive en dansant et se jette sur la première encore au sol, elle mime alors l'acte sexuel en se mettant à califourchon sur la première ou en s'allongeant. La danse "dégénère » alors. Les femmes s'entassent les unes sur les autres, par trois, par quatre, par « paquet »... Dans ce cas, le joueur de tama, dans une feinte colère, intervient pour arrêter cette frénésie générale, et toutes retournent s'asseoir rapidement. Par sa plus grande mobilité, il est celui qui semble être le plus proche des danseuses. Même s'il n'y a pas souvent de réel contact physique entre les musiciens et les jeunes femmes, j'ai pu observer quelquefois un percussionniste pincer ou caresser une fesse furtivement.

La seconde réunion, toujours dans une petite cour du quartier de la Médina, débute également vers 17 heures. On compte une trentaine de jeunes femmes avec leurs bébés et quelques petites filles. Les musiciens sont cinq (sabar et tama). Les femmes ne sont pas spécialement habillées pour l'occasion et portent apparemment des tenues de tous les jours. Les corps se libèrent au fur et à mesure des rythmes et au paroxysme de la fête, une jeune femme soulève son pagne pour effectuer un saut, tout en montrant son string en dentelle, décoré de mèches de cheveux, de tablettes de chewing-gum et de bonbons à la menthe. De la même manière, une seconde jeune femme dévoile sa culotte sur laquelle est cousue, sur le devant, une rose rouge en tissu. Au plus fort de la séance du lëmbël, une troisième lève son pagne et montre aux autres femmes, un sexe d'homme en plastique accroché à sa taille par une ficelle. Elle joue avec, l'attrape, le place devant les autres danseuses en simulant les élans d'un homme pressé, ce qui provoque l'hilarité générale pendant quelques minutes.

\section{«Travailler le corps » : soins et parures}

$\mathrm{Si}$, lors de cette deuxième séance, les femmes ne portent pas d'habits de fête, elles n'en ont pas moins soigné leurs « dessous ». Dans la danse, le vêtement forme quasiment une seconde peau que la danseuse utilise pour évoluer dans sa performance et embellir certains mouvements. Le fait de porter un boubou ou un jean n'offrira pas la même perception des mouvements dansés, des formes du corps, de ce qu'on laisse voir ou de ce que l'on cache. Les vêtements et les parures sont porteurs de significations et font partie des éléments qui

16. Dans le sabar, il existe plusieurs rythmes sur lesquels les danseuses évoluent, dont voici les principaux : le faru jar, le ceebujen, le baara mbaye, le kaolack, le mbabas, le niari goron et le lëmbël.

17. Ce terme désigne le joueur de tama qui dirige l'ensemble de ces petits tambours d'aisselle. Le rythme du ventilateur est toujours accompagné par des tama. 
donnent au sabar son caractère sensuel et érotique. Pour une fête, le plus souvent, les femmes et les jeunes filles préfèrent revêtir le vêtement «traditionnel », et de nombreuses formes de boubous sont représentées, ainsi qu'une multitude de tissus et de matières : le bazin (coton damassé), le lin, la «djitpur» (wolofisation de « guipure »), le tiup (tissu teint à l'indigo), le « Khartoum » (tissu en voile de coton très léger, porté par les mauritaniennes) et le boubou «takk-uwé » (tissu transparent en voile ou en mousseline).

Le pagne se dit pendal en wolof. Aujourd'hui, les pagnes tissés, très lourds et très chauds, sont la plupart du temps remplacés par des pagnes «seur-u-sor » (constitués de bandes de coton léger) pour l'habillement. Ils restent malgré tout plus importants que les autres, notamment pour faire des cadeaux, envelopper le bébé (le pagne s'appelle alors mbotu) et couvrir les morts. Les femmes peuvent porter plusieurs pagnes sous leur boubou, cela s'appelle këfëlu (le fait de mettre plusieurs pagnes pour augmenter la rondeur des fesses). L'importance du pagne dans la danse est explicitée par Sokhna Fall dans les termes suivants : il « rappelle à la danseuse la vie de son corps, collant sur les reins, obligeant sur les cuisses, incertain à la taille, il donne la parole au bas du corps » (1998 : 87).

Sous ces pagnes, les femmes portent de petits pagnes connus sous le nom de beeco. Il en existe de très nombreuses sortes, qui connaissent des phénomènes de mode ${ }^{18}$. Parmi les beeco, on trouve notamment le «ne-degueur », littéralement " ce qui est dur ", il est fabriqué à partir d'un tissu que l'on décore en faisant des trous à l'aide d'un ciseau (en forme de petits losanges). Quant au «bine-bine », « vas-y doucement », il est fait dans un tissu en satin, décoré avec des petits trous obtenus avec un morceau de bois brûlant. « Les papiers de la maison » est un pagne qui ressemble au « filet ». Il est décrit comme ayant le pouvoir d'envoûter les hommes. Quand une femme porte ce type de pagne, elle ne demande pas à son époux un téléviseur ou un frigidaire, mais le titre de propriété de la maison et celui-ci est censé ne rien pouvoir lui refuser, envoûté par les charmes de sa femme, mis en valeur par ce « filet ».

Il existe également des petits pagnes brodés ou peints qui mettent en scène des positions sexuelles, des vulves et des pénis, ainsi que des petites phrases comme «saf mana dem, saf, saf, saf» (« quelque chose de bon, de délicieux »). De tels beeco se nomment diigal, qui signifie « s'immerger », " aller au fond et y rester un moment», au sens sexuel du terme. Ces petits pagnes sont destinés au mari, au fiancé ou à l'amoureux, ils relèvent donc de l'intimité. Pourtant, dans un sabar, les danseuses relèvent parfois leurs pagnes pour laisser voir ces beeco. Elles montrent ce qui normalement est caché. Cette démonstration de l'intime plaît particulièrement aux percussionnistes. Tous m'ont parlé de ces fameux pagnes «érotiques ", qui sont censés leur donner du courage et de la force pour jouer et faire durer la fête.

En plus de ces vêtements, les femmes utilisent d'autres éléments pour se faire belles. Ces secrets dévoilés par certains mouvements dansés sont des objets quasiment "incorporés ", tant ils symbolisent le pouvoir de séduction des femmes sénégalaises. Elles imprègnent leurs

18. De nombreux termes pour désigner ces petits pagnes sont inventés sans cesse et sont souvent inspirés de la musique sénégalaise moderne appelée mbalax en wolof et très à la mode dans tout le Sénégal, dont les paroles évoquent souvent la sexualité et les sentiments amoureux. 
vêtements et leur corps de cuuraay, encens sénégalais ${ }^{19}$ composé d'encens, de bois de santal, de gomme arabique et de parfums. Elles attachent à leurs sous-vêtements des petits sachets appelés gongo, composés de musc, de santal, de parfums et d'encens. Elles massent leur corps avec du karité, qu'elles ont fait fondre préalablement, pour ajouter, une fois que le beurre est devenu liquide, le parfum de leur choix. Les parures de bijoux dorés et argentés sont complétées par des colliers de taille, appelés bine bine, djaldjali ou fer (fabriqués avec des perles, en pâte de verre parfumée). Les perles peuvent être également en plastique, ou en rocaille aujourd'hui. Le sens de l'odorat est donc particulièrement sollicité par toute cette préparation et cette mise en beauté.

Les perles des colliers de taille des femmes appartenant au groupe des boisseliers (lawbe ${ }^{20}$ sont réputées porter chance et protéger celui qui en détient. Ainsi, selon un musicien : « Si une lawbe, qui vient de danser, casse un fer, il faut ramasser les perles qui tombent et les garder avec toi car ça porte bonheur, mais seulement si ce sont des fer de lawbe $»^{21}$. Ce pouvoir sexuel conféré aux femmes peut également s'avérer nuisible pour l'efficacité de certaines amulettes (teere) ${ }^{22}$. Un autre musicien me signalait d'ailleurs à ce propos :

Il y a des choses qu'il ne faut pas faire quand on porte certains teere. Par exemple, il $y$ en a que tu ne dois pas porter quand tu fais l'amour, sinon ils se cassent et ils n'ont plus d'efficacité. C'est pareil pendant la danse, quand une danseuse danse devant toi de manière trop provocante, et que tu vois trop de choses, ça peut casser le grigri. ${ }^{23}$

Mais si parure et apprêts sont des éléments essentiels de la performance, ils ne font évidemment sens que dans leur mise en mouvement.

\section{Le langage de la danse : images et symboles de la sexualité dans le sabar}

Quelles sont les caractéristiques si particulières du sabar? Même si chaque rythme est constitué de mouvements spécifiques, l'élément commun qui caractérise le sabar est le saut, appelé cinq temps ou ndior en wolof. Les rythmes qui accompagnent la danse oscillent en permanence entre une pulsation binaire et une pulsation ternaire. Cette dynamique très

19. Chaque nouveau cuuraay vendu sur le marché, mélange subtil de différents parfums, porte un nom spécifique très souvent évocateur comme «ne bouge pas chéri » ou « tu es mon âme ». Les femmes en achètent souvent, mais elles peuvent également se confectionner des senteurs personnelles et uniques.

20. Les lawbe sont des boisseliers. Les hommes travaillent le bois et fabriquent notamment les fûts des tambours, tandis que les femmes ont la réputation d'être les spécialistes des choses liées à la sexualité. Elles confectionnent de nombreux objets vendus sur les marchés, comme les petits pagnes, les colliers de taille odorants, les encens spécifiques aux pouvoirs envoûtants, et également de nombreuses potions à partir de substances variées qui agissent notamment pour rétrécir le vagin avant un rapport. En voici deux exemples : « la glace », poudre blanche à mélanger avec du lait et à boire trente minutes avant l'amour, et « le curedent », il faut commencer à se curer les dents en fin d'après-midi (tout en avalant la salive) pour que l'effet attendu soit effectif le soir. Les femmes lawbe ont également la réputation d'exceller dans l'art du lëmbël, dont elles seraient même les inventrices.

21. Entretien avec un griot-percussionniste, Dakar, janvier 2007.

22. Un teere est une amulette confectionnée par les soins d'un marabout. Il est censé protéger la personne qui le porte de différentes formes d'attaque, notamment du mauvais œil, des dangers des paroles malveillantes et de la sorcellerie.

23. Entretien avec un griot-percussionniste, Dakar, janvier 2007. 
particulière semble créer un équilibre instable qui se retrouve dans les pas du sabar. Le temps dansé est très bref, on reste rarement plus de trente secondes au centre du cercle. On peut dire qu'il existe deux styles : le sabar aérien, constitué de sauts dirigés vers le haut et le sabar près du sol. En wolof, le fait de danser de manière aérienne se nomme "fecc gaaw » qui signifie littéralement « danser rapidement » et danser près du sol se nomme «fecc suuf», signifiant « danser dessous ».

Toute particulière est la danse du « ventilateur », évoquée plus haut. Plusieurs types de rythmes de clôture (lëmbël) sont joués par les musiciens. En voici quelques-uns, le plus souvent tirés de morceaux de mbalax, musique sénégalaise moderne très appréciée en contexte urbain ${ }^{24}$ : le « lëmbël naar », le «fuil meti », le « climatiseur », le « vibreur » et le «bosël». Le premier signifie littéralement « ventilateur des Arabes » et les mouvements imitent ceux de la «danse orientale $»^{25}$. Les danseuses effectuent des mouvements de hanche saccadés, tout en avançant vers les musiciens, les bras et les poignets ondulant comme des serpents. Le second signifie « là où tu as mal », le mouvement principal reste la rotation des fesses tout comme le « climatiseur ». Le « vibreur» annonce, quant à lui, un mouvement particulier qui consiste à rester immobile et à contracter en alternance les muscles de la fesse gauche et de la fesse droite, en suivant le rythme du tambour d'aisselle. «Bosël» signifie appuyer. Accompagné d'un court texte psalmodié (taasu ${ }^{26}$, ce rythme annonce également un mouvement spécifique. Il se danse souvent à deux personnes, l'une en face de l'autre. La particularité de ce lëmbël est la suivante, quand le musicien chante « bosël, bosël », les deux personnes qui se font face donnent deux coups de rein en direction l'une de l'autre, tout en se tenant par les épaules. Toutes ces manières de danser le « ventilateur» sont des modes et de nouvelles s'inventent sans cesse. Le rythme effectué par les musiciens reste sensiblement le même, ce sont les paroles qui changent. Certains mouvements spécifiques viennent également se rajouter au « traditionnel ventilateur». Quand les tamas entament ce rythme, l'excitation est à son comble et toutes connaissent les dernières phases dansées, notamment par l'intermédiaire du mbalax et des tubes musicaux, où les danseurs ne cessent d'inventer des nouveaux pas qui deviennent très vite les mouvements à la mode qu'il faut savoir reproduire.

C'est à l'aune de trois qualités que l'on reconnaît une bonne danseuse de sabar. Ainsi faut-il qu'elle mette dans sa danse :

- du « bëret » (« se lever brusquement »), on peut également employer le terme «xadar» (« tempérament bilieux »),

24. Le lëmbël est un rythme « traditionnel » qui se nourrit constamment de la musique moderne sénégalaise. C'est notamment le percussionniste et chanteur Mbaye Dieye Faye qui, avec Salam Diallo et Pape Ndiaye Thiopet, crée les morceaux de mbalax les plus " hot », comme « bine bine tuuti » qui signifie "vas-y doucement » et qui est aussi le terme pour désigner les colliers de taille, ainsi que "Ubil barken Ndiaye » traduit par « ouvre-moi ton minou ». Lëmbël naar, la danse des Arabes en référence aux habitants de la Mauritanie, est tirée d'un clip du chanteur Secka. Ce terme se traduit aussi bien par " Arabe » que par « Maure » d'après le dictionnaire de Jean-Léopold Diouf (2003 : 233).

25. Terme trop générique pour être significatif, mais je l'utilise car les mouvements du « lëmbël nar » apparaissent comme une caricature de ce qu'on appelle communément la « danse du ventre ».

26. Taasu en wolof désigne un court texte chanté ou psalmodié. Dans cet exemple, le percussionniste utilise l'homorythmie pour renforcer l'effet recherché : il déclame le taasu tout en le jouant sur son tambour. 
- du « cekete » (certains utilisent les termes «nokkos» littéralement « piler les épices » ou encore «safal » qui signifie « qui est bien relevé » et « qui a de la saveur »),

- du « daanel» (« conclure »).

La première qualité symbolise l'énergie et la puissance. La seconde, " cekete », est également utilisée quand on cuisine et qu'on rajoute des épices pour donner du goût au plat. Ce sont les petites attentions que rajoute la danseuse pour apporter le côté pimenté, osé et érotique de sa danse : un regard, un mouvement de boubou, bref, tout ce qui personnalise sa prestation. Les métaphores liées au vocabulaire culinaire sont très fréquentes pour qualifier la danse et notamment l'aspect sexuel qui caractérise les mouvements. De telles métaphores sont d'ailleurs également présentes dans le langage de la sexualité. Enfin, la troisième caractéristique repose sur la fin de la danse, la manière de couper et de terminer sa performance. C'est un aspect très important dans l'appréciation du sabar.

Certains chants qui accompagnent les danses sont des métaphores culinaires de la sexualité insistant sur les condiments qui donnent du goût. En voici un exemple :

Sa data neex a neex a neex ... poobar, kani, soble, jimbo, vinegar, netetu, waaye sama kooy a ko daq, moom mi genne meew, meew tangana, meew gloria, meew nestle.

Ton sexe est si bon..., poivre, piment, oignon, jimbo, vinaigre, netetu (condiment, graines de néré fermentées), mais mon pénis est meilleur, lui il sort du lait, du lait chaud, du lait Gloria, du lait Nestlé.

Notons également qu'une bonne performance dansée sera qualifiée de «fecc tooy », cet adjectif signifie « être mouillé » en wolof. Une danse mouillée est une danse particulièrement réussie et $"$ sexy $»^{27}$. Cet adjectif est également utilisé pour qualifier une jeune fille qui suscite le désir.

D’autres sont clairement des chants licencieux ${ }^{28}$ qui célèbrent la puissance masculine et la virilité :

Data bu nuux baaxul dëngal kooy

Pan pa la la tan, Pan pa la la tan

Kaay ma katala yo naari bum yu gaaw

Si ton sexe n'est pas droit, ce n'est pas bon car ça tord le sexe de l'homme.

Viens que je te baise, j'ai deux manières de baiser rapidement.

\section{Les interactions du sabar}

Attachons-nous un instant au rapport particulier qui se construit à partir du dialogue entre la danseuse qui entre en scène et le musicien qui possède la fonction de tambour-major. Cette interaction, tissée par un échange réciproque d'énergie entre les mouvements dansés et le jeu du tambour, est au cœur de la réussite de la performance. Cette rencontre est très souvent soulignée dans les entretiens menés avec les musiciens :

27. Cette traduction m'a été donnée par un percussionniste lors d'un sabar en région parisienne en 2008.

28. Les termes appartiennent à un langage familier dont la traduction n'est pas aisée dans la mesure où ces chants sont construits à partir d'images et de métaphores. Un percussionniste, C. Mboup, m’a aidé à traduire ces paroles à Dakar, en janvier 2007. 
Les danseuses, elles doivent faire avec le feeling, tu dois avoir du sucre, du sel, des fleurs, du jimbo, tout ça quoi. C'est pour dire que c'est le style qui est important. C'est vraiment un duo. Il ne faut pas rester timide dans la danse sinon le griot ne reçoit rien et il s'ennuie. Si une danseuse sort et [que] tout de suite une autre enchaîne, c'est bon, c'est ça le dialogue. ${ }^{29}$

Aujourd'hui, le sabar c'est beaucoup plus rapide qu'avant et donc beaucoup plus fatigant qu'avant à jouer. La danse est beaucoup plus sexy qu'avant. C'est joli et ce n'est pas une danse réservée. Moi, la danseuse qui danse mais qui ne me montre rien, ça ne me donne pas le courage de jouer. Nous on joue pendant longtemps sans pause, on se fait mal aux mains, on se tape sur les doigts, on a besoin de courage pour trouver cette force. ${ }^{30}$

Aujourd'hui en effet, le discours commun souligne souvent la perte d'élégance de la danse au profit de sa « sexualisation ». Les arguments évoqués par les acteurs sont multiples, même si deux reviennent souvent, à savoir tout d'abord l'influence des clips américains de RAP et de R\&B, qui mettent en scène des filles dénudées et des chorégraphies lascives, et ensuite une certaine «masculinisation » du sabar: les hommes, de plus en plus nombreux à devenir des danseurs professionnels (voir Neveu-Kringelbach 2007), envahissent les sabar de rue et excellent dans cet art en offrant un style acrobatique. Les filles, ne pouvant atteindre cette dextérité et cette force physique, n'ont que l'évocation de la sexualité pour se faire remarquer durant leur performance. Tous ne partagent pas toutefois ce point de vue, et les références à la sexualité dans le sabar n'ont par ailleurs rien de nouveau (voir supra note 6).

En fait, la danseuse doit parfaitement connaître et comprendre les différents rythmes du sabar pour assurer une bonne performance. Ce moment de danse est vécu comme un échange et une construction à deux, entre musicien et danseuse, où la circulation d'énergie se fait par l'envie du musicien de donner sa force et par sa capacité à transformer les accents du corps en coups de baguette. Ce désir semble naître de la qualité de la performance dansée (le « feeling » ou le ressenti de la danseuse) qui s'évalue, selon les musiciens, au niveau de la dextérité, de la séduction et de la précision qui émanent des mouvements qu'elle réalise. La danseuse utilise son corps pour communiquer et lancer les appels, le musicien se sert de son tambour pour y répondre. L'énergie nécessaire à la danse se transmet par le biais du corps et du tambour, ils sont à la fois diffuseurs d'énergie et en même temps réceptacles.

Cet échange prend une autre dimension quand le joueur de tambour d'aisselle, le tamakat, entre en scène. C'est l'évènement qui marque le paroxysme du sabar aux niveaux de l'ambiance, de la mise en scène de la sexualité et de l'échange interactionnel entre le musicien et la danseuse. Entre ces acteurs se noue une relation dont le rythme et l'intensité ne sont pas sans évoquer une sorte de copulation symbolique. Durant une performance de « ventilateur », l'union peut prendre une forme plus concrète : le musicien, qui évolue avec son tama au centre du cercle de danse, peut, d'un geste rapide, toucher la danseuse avec un mouvement vif de bassin, symbolisant l'union sexuelle. Durant une séance de « ventilateur », l'échange d'énergie et l'union symbolique entre le musicien et la danseuse, ne passent plus par le tambour, mais par un corps à corps très bref, les deux étant en contact l'espace d'un

29. Extrait d'un entretien réalisé à Paris avec M. Samb, griot percussionniste, en avril 2008.

30. Extrait d'un entretien réalisé à Paris avec M. Diop Mbengue, percussionniste, en avril 2008. 
instant. Le tamakat, en touchant rapidement la danseuse de manière significative veut la féliciter et la remercier pour la qualité de sa prestation. C'est une façon de lui montrer qu'il a été touché et qu'il s'est amusé à accompagner ses mouvements. C'est aussi un moyen de réinvestir l'attention du public et de tourner tous les regards vers lui. Au cœur de l'exhibition dansée, le joueur de tama répond par une gestuelle stéréotypée et attendue dans toute fin de sabar. C'est un jeu au sein de la danse, mais un jeu qui ne peut exister qu'en tant qu'il signe une véritable performance artistique. Le tamakat sait exactement les gestes qu'il peut se permettre d'effectuer auprès d'une danseuse et connaît les limites à ne pas franchir, les règles à ne pas transgresser. Le contact doit être bref et joué magistralement. Dans ce cas, il est accepté par tous. Cette action n'est pas le simple reflet de l'état émotionnel dans lequel se trouverait le musicien face aux manifestations lascives des danseuses, c'est un geste attendu par toutes, conforme aux règles du jeu de la danse du ventilateur. Cette mise en scène de l'acte sexuel ne met pas réellement en relation les acteurs présents, On se sert du partenaire (le musicien ou la danseuse) pour jouer à un jeu qui ne s'adresse pas (seulement) à lui. Le sabar mobilise des relations plus complexes que ce qui est immédiatement donné à voir. En effet, le but n'est pas de faire des conquêtes, il n'y a pas de corrélation avec la vie quotidienne, on séduit, mais ce n'est pas une technique de «drague ». C'est un jeu qui reste cantonné à l'espace de danse. Notons que les acteurs jouent la sexualité mais sans risque de débordement car socialement, les hommes présents sont ceux avec qui les relations sexuelles sont interdites, les griots étant des hommes «castés $»^{31}$. On séduit, on met en scène des métaphores et des images de la sexualité avec des partenaires, qui, par définition sociale, n'en sont pas (les autres femmes présentes et les griots).

Par contre, ce qui semble s'échanger, c'est le savoir technique du rythme et de la danse, ainsi que le plaisir de construire quelque chose de beau, ensemble. Finalement, cet échange d'énergie et cette osmose entre musicien et danseuse permettent à chacun de briller individuellement, toujours dans la recherche d'exhibition.

\section{«Apprendre par corps " ${ }^{32}$}

À propos de l'éducation sexuelle des jeunes filles au Sénégal, Colette Le Cour Grandmaison rapportait qu'il n'y avait pas d'explication donnée sur la sexualité dans le cadre familial, où "l'ignorance apparaît comme une protection assurée de la virginité ». Elle ajoutait toutefois que, comme cela s'observe toujours aujourd'hui dans de nombreuses familles dakaroises, « les couples partagent souvent la chambre avec les enfants », lesquels développaient de ce fait « une connaissance rapide de l'intimité conjugale ». Cependant, en raison du " silence total » pesant entre mères et filles sur la question de la sexualité, « les adolescentes doivent feindre d'ignorer les rencontres entre femmes (où elles donnent libre cours à leur sexualité) alors qu'elles en sont les témoins illicites mais tout de même présents » (1967: 58).

31. Les griots jouent un rôle dans l'éducation sexuelle. Cette délégation des tâches pédagogiques s'expliquent par le fait qu'ils détiennent une liberté de parole et d'actions que ne possèdent pas les «nobles ». Ceux-ci doivent en effet respecter un code de l'honneur qui évacue presque complètement la possibilité de parler en société d'activités liées au sexe.

32. D'après le titre de Sylvia Faure (2000). 
En fait, comme je l'ai observé, les enfants, dès le plus jeune âge, ne sont pas seulement témoins, mais également acteurs, invités à reproduire les mouvements dansés des adultes. Les moments de sabar représentent pour les enfants des deux sexes, une réelle initiation aux jeux de séduction et une mise en scène d'actes et de paroles référés à la sexualité. Les tout petits et les jeunes filles sont tolérés dans les moments de danse entre femmes. Ils sont sollicités pour s'initier à la danse et les jeunes filles, même si elles doivent rester passives devant les aînées, organisent des moments qui leur sont dédiés pour s'exprimer de la même manière. Si la danse semble participer à la transmission de certaines représentations liées à la sexualité, on peut néanmoins se poser la question suivante : ces enfants et ces jeunes filles n'en seraient-ils pas finalement les destinataires principaux, ou tout au moins souhaités, à l'instar de la position qu'ils occupent dans d'autres manifestations, telles les séances de contes ? Ainsi, Suzanne Lallemand, dans son ouvrage sur les contes paillards d'Afrique de l'Ouest, rappelle que ces récits sexuels qui existent dans plusieurs sociétés, sont avant tout des récits pédagogiques. Selon elle, «non seulement les jeunes enfants constituent un public toléré, admis à la réception de messages sexuels délivrés par certains récits, mais il en est même la cible principale, l'auditoire électif, le destinataire souhaité » (1985 : 15-16).

Dans le cadre de réunions féminines, les mères exercent les petites à la danse. Celles qui ne marchent pas encore sont allongées sur les genoux d'une adulte qui pose alors ses mains sur les fesses de la novice pour les faire bouger en rythme ${ }^{33}$. Une fois que les petites marchent, l'apprentissage est basé sur l'imitation. Sylvia Faure note que chez les jeunes enfants, l'imitation se rapporte d'abord au mimétisme (défini comme étant un processus d'acquisition pratique reposant sur le faire semblant et supposant un rapport global d'identification), les petits ayant une tendance naturelle à se modeler sur autrui (2000:124). Pierre Bourdieu, s'appuyant sur ses observations en Kabylie, remarque que l'essentiel de la transmission peut s'effectuer par la pratique sans accéder au niveau du discours (notamment Bourdieu 1972 : 189). Dès la plus tendre enfance, au Sénégal et en contexte migratoire, de nombreuses fillettes sont, de ces deux manières, entraînées à la réalisation des mouvements du lëmbël. À l'extérieur, pendant ce temps, les plus grands, qui ne peuvent rentrer, dansent sur les tambours dont le son sort largement de l'espace clos et organisent leur petit sabar. Les mouvements qu'ils effectuent sont déjà très imagés et reprennent à l'identique ceux des adultes, comme un savoir incorporé, intégré très tôt. Lors d'une participation à une tontine, se déroulant dans une petite cour intérieure, j'ai relevé la présence de nombreux jeunes garçons grimpés sur les toits voisins pour voir ce que faisaient les femmes. Ils dansaient eux aussi en produisant des mimiques et des gestes de bouffonnerie. Les femmes semblaient s'en moquer et n'y prêtaient pas attention. Cet autre exemple illustre des modalités d'éducation sexuelle ne passant pas par un enseignement dogmatique, mais davantage par le corps, par l'intermédiaire de la danse et du mimétisme. Les chants qui accompagnent le sabar entrent également dans le champ de la socialisation sexuelle puisqu'ils sont des évocations explicites des organes génitaux et de l'acte sexuel.

33. De la même manière, Jacqueline Rabain rappelle que toutes les opérations rituelles de l'enfance soulignent l'investissement collectif du corps et son marquage social. Par exemple, le massage forme et modèle le corps du petit enfant selon des caractéristiques sexuelles : il durcit le corps du garçon et épanouit les fesses des filles $(1979$ : 81). Il semble en être de même pour le sabar où la pratique des tambours muscle les bras et le torse des musiciens, tandis que la danse façonne les fesses et assouplit les muscles des danseuses. 
Par ailleurs, des sabar d'enfants se tiennent souvent dans les quartiers populaires. Quelques musiciens sont payés pour animer et un petit cercle de chaises est installé. Les fillettes évoluent sur les mêmes rythmes que les adultes et reproduisent les mouvements des aînées. Elles sont habillées à l'image de ces dernières. En effet, une tenue est confectionnée à leur intention, reproduction parfaite d'une coupe à la mode : ndoket ou boubou, mussor (mouchoir de tête), petit pagne, ... Quelques adultes sont présentes, mères ou grandes sœurs, pour encourager les petites à se lancer dans le cercle. Il existe également des tontines de jeunes filles adolescentes qui se déroulent également autour de danses accompagnées de percussionnistes, à la manière des réunions de femmes mariées ${ }^{34}$.

La danse est donc présente à tous les âges de la vie d'une femme wolof et cela dès la plus tendre enfance. Elle représente un moyen d'apprendre, de s'entraîner, de laisser exprimer sa féminité, de s'exercer aux techniques de séduction et d'intégrer des éléments de la sexualité. Solliciter les petites et les jeunes filles pour venir danser, que ce soit dans le cadre d'une réunion féminine ou d'un sabar est une pratique courante. Il n'est pas rare qu'une jeune fille assurant une performance réussie et remarquée se voit offrir, par les femmes de l'assistance, une paire de chaussures à talons, un sac à main, un string, ... Soit une série d'objets qui symbolisent la féminité, la sexualité et le pouvoir de séduction mis en avant comme une force.

Les arguments exposés par Suzanne Lallemand, reprenant l'analyse de M. Duponchel concernant les contes baoulés de Côte d'Ivoire, peuvent s'appliquer au sabar:

Certains contes racontés à la veillée familiale sont très instructifs sur les choses du sexe et traduisent la nature avec lequel ce domaine est présenté aux enfants qui à tout âge bénéficient de cet enseignement par la tradition orale. [...] Rien n'est enseigné à l'enfant de façon dogmatique, mais on lui laisse puiser librement dans le vécu quotidien des adultes. (1985: 19)

Cependant, ce mode pédagogique qui repose sur la parole ne semble pas totalement se confondre avec celui que propose la danse. En effet, concernant les contes paillards, S. Lallemand précise qu' "il est beaucoup de choses que ces contes ne disent point », et qu'en aucun cas, "l'ensemble de ces contes ne peut évoquer un de ces manuels à recettes susceptibles d'indiquer au néophyte la manière de varier ses futurs plaisirs ». Qu'en estil des mouvements du sabar qui miment l'acte sexuel, du fait de montrer son sexe dans la danse, de la présence symbolique du sexe masculin par l'intermédiaire d'un phallus façonné en plastique ou en bois, du jeu entre le joueur de tama et la danseuse simulant une copulation? Les enfants présents sont ainsi témoins de mises en scènes qui évoquent la sexualité et plus particulièrement l'acte lui-même. Si le sabar entre dans un modèle éducatif au même titre que les contes paillards autrefois, que propose-t-il de manière implicite et symbolique ? Je pense que si le conte suggère, la danse mime et imprime une représentation de l'acte sexuel par la monstration du corps et par la gestuelle qui prend parfois la figure d'une restitution fidèle.

34. Jacqueline Rabain décrit un jeu similaire : le baptême de poupée, image de la cérémonie adulte d'attribution d'un nom à l'enfant au $8^{\mathrm{e}}$ jour. La cérémonie est un repas de fête consommé par les enfants de 8 à 12 ans d'un quartier du village. L'auteur explique que ce jeu permet aux enfants de faire l'apprentissage de certains rôles sociaux qu'ils se distribuent; dont ceux des griots hommes et femmes, et de les articuler à travers les activités de préparation culinaire et de danse (1979: 51). 
La danse semble aussi constituer un moyen d' " éduquer » certaines femmes adultes, mariées mais trop timides et réservées, ainsi que l'illustre cet extrait :

Il y a des femmes qui savent s'y prendre avec leur mari, et d'autres qui sont timides, complexées quoi. Dans nos fêtes, les femmes dégourdies montrent tout ce qu'elles font pour leur mari. Elles encouragent les femmes timides et les poussent au milieu. On les oblige à danser. On leur parle et, petit à petit, elles vont changer. (Fall 1998 : 118)

Il n'y a donc pas d'âge pour apprendre et appliquer « des recettes » qu'offre le sabar à toutes celles qui y participent ${ }^{35}$, pour séduire.

\section{Le sabar: une affirmation des codes sexués}

Le sabar célèbre les représentations de la féminité et de la virilité. Les femmes doivent se montrer séduisantes et excitantes lors de leur performance. Les métaphores de la sexualité sont les éléments principaux auxquels elles recourent et constituent les critères qui seront jugés durant leurs prestations. Les hommes qui, aujourd'hui, sont acceptés sur la piste d'un sabar, le sont en raison de leur statut particulier, celui de professionnel de la danse ${ }^{36}$. Plus généralement les hommes danseurs ont développé un style qui leur est propre et qui n'engage pas les mêmes attributs que ceux des danses féminines. Les hommes excellent dans l'invention de multiples « phases ». Ils insistent sur l'aspect théâtral dans leurs réalisations à travers le mime pour se faire remarquer. Ici, ce n'est pas la sensualité qui est recherchée mais la démonstration de force, de puissance musculaire, d'acrobaties, et la capacité à faire rire le public par des gestes qui suggèrent des situations comiques. La pratique de la danse par des hommes est ainsi acceptée parce qu'elle incarne une image de la masculinité conforme aux codes de la virilité. Car un homme qui, en dansant, exprime une certaine féminité, sera stigmatisé, traité de "goor-jigeen $»^{37}$ (homosexuel) et rejeté par la majorité de l'assemblée au regard d'une aversion généralisée de la société sénégalaise pour l'homosexualitée ${ }^{38}$. Il existe donc, des limites à ne pas franchir et des codes à respecter qui inscrivent chaque sexe dans des attitudes corporelles et dans des «canons » respectifs.

Cependant, en exhibant crûment une mise en scène de l'acte sexuel qui met à mal toutes les règles de la vie quotidienne, le sabar joue aussi sur le registre de l'obscénité,

35. Il faut souligner une certaine immixtion sociale dans l'intimité conjugale à travers l'enseignement sexuel que les épouses reçoivent par l'entremise des proches parentes (cousines, tantes) et des amies. L'érotisme conjugal n'est pas qu'une histoire de couple et la danse est un moyen de rappeler les bonnes conduites gestuelles et une certaine soumission au désir de l'homme.

36. Sur la danse professionnelle, lire l'article d'Hélène Neveu Kringelbach (2007 : 81-101).

37. Littéralement un « homme femme ». Des figures traditionnelles d'hommes danseurs travestis existent dans la société wolof et tenaient autrefois un rôle important dans les cérémonies, mais aujourd'hui, ils sont très mal considérés. Certains groupes de danse tentent encore d'apparaître dans les sabar déguisés en femmes, mais ils sont très vite hués et sommés de partir.

38. La société urbaine dakaroise connaît un durcissement à l'égard de l'homosexualité. De nombreux faits exposés dans la presse sénégalaise relatent des passages à tabac d'homosexuels (cas de violences très grave, profanation de tombes, durcissement des peines infligées par la justice à l'encontre d'homosexuels). Les prêches de certains dignitaires religieux sont des plus virulents, exposant l'homosexualité comme un mal à éradiquer, une maladie contagieuse qui s'empare de la jeunesse. Au Sénégal, l'homosexualité est officiellement interdite et passible, selon le Code pénal, d'une peine allant d'un à cinq ans d'emprisonnement. 
manifestation attentatoire à la pudeur ordinaire, que l'on pourrait comprendre, selon les termes de Beidelman (1968 : 115), à partir de la catégorie de la nakedness, à savoir comme « a state of being undressed which causes shame, disrespect, and harmful results in one's social surrounding ». Evans-Pritchard fut l'un des premiers anthropologues africanistes à tenter de comprendre le sens des comportements d' " obscénité collective ». Examinant de près un ensemble de chants et de pantomimes licencieux, le plus souvent réalisés par les femmes dans quelques sociétés de langue bantoue, il rappelait qu'en certaines circonstances, ces comportements ne sont pas seulement autorisés, mais prescrits. Il leur attribuait une double fonction, une fonction générale, celle de mettre en évidence la valeur sociale de l'activité avec laquelle elle est liée, et une fonction de décharge émotive : l'obscénité s'exprime lors d'occasions chargées d'une grande tension émotionnelle et fournirait alors « un moyen socialement autorisé d'exprimer cette émotion » (Evans-Pritchard 1971). On sait, par ailleurs, que de tels comportements obscènes sont fréquemment mobilisés lorsqu'il s'agit, dans les sociétés et les époques les plus diverses ${ }^{39}$, de faire face à différentes formes de danger ou de calamité. En Afrique, les occurrences les plus fréquemment mentionnées sont la guerre, la sécheresse et les épidémies ${ }^{40}$. Ces contextes n'ont certes rien à voir avec celui du sabar, pourtant, l'exhibition et la mise en scène de la sexualité y sont sinon prescrites, du moins fortement attendues. D'après Michael Houseman reprenant l'argument de Marcel Mauss (1921), « l'expression obligatoire des sentiments » lors des rites consiste moins en l'exhibition convenue de certains états émotionnels, qu'en l'impératif de s'engager dans certaines activités dont la poursuite implique des manifestations affectives (Houseman 2008 : 8). Dans le sabar, chacune des participantes se doit de proposer une surenchère de mouvements osés, de corps déshabillés et d'obscénité qui permet une décharge des tensions. Elle est l'expression collective d'émotions déclenchées dans cet espace de liberté, d'actes et de paroles que les femmes ne semblent pas avoir dans le quotidien. Mais que chercheraientelles à conjurer?

Du fait de son aspect « hyper-sexualisé », la danse wolof a pu être perçue comme une forme d'exutoire face à la domination masculine.

La danse, semble être une expression de résistance qui révèle quelque chose concernant le peu d'autonomie des femmes dans les politiques de genre au Sénégal en milieu urbain. [...] Les résistances quotidiennes ne mettent pas seulement en évidence l'action d'une critique consciente des membres subordonnés à une culture, c'est également un moyen de comprendre les différentes formes de pouvoir qui englobent les notions de résistance, de complicité et de structure de domination. (Heath 1994 : 88)

39. H. P. Duerr a mené une exploration encyclopédique de ces exemples où les femmes, que ce soit pour exprimer le plus grand mépris, effrayer ou insulter l'ennemi, raffermir leurs guerriers, faire rire les dieux ou encore assurer le renouvellement de la fertilité, usent de l' " arme » qui est la leur : dévoiler et exposer leur sexe. Ce geste, dit-il, on le trouve dans des cultures et des époques si diverses que l'on est tenté de le qualifier d'universel (Duerr $1992: 105$ ).

40. Ainsi, pour ne prendre que des exemples de sociétés proches, chez les Sereer, les pantomimes érotiques des femmes exécutées dans le cadre des rites d'appel à la pluie, auraient pour but « de dérider Dieu et les puissances ancestrales, pangol » (Dupire 1976). Chez les Joola, les comportements d'obscénité féminine sont intégrés à un rituel réservé aux femmes en mal d'enfants (Journet 2008). 
Si l'auteur relativise sa position, signalant aussi que «la dynamique est largement plus complexe qu'une simple résistance des femmes face à leur subordination envers les hommes », elle n'apporte pas d'autre interprétation à ce qui est en jeu.

Cependant, peut-on réduire l'analyse du sabar à une forme rituelle de conjuration de la domination masculine, ou encore à une attitude ostentatoire de compétition entre participantes, au risque de s'inscrire dans une approche fonctionnaliste et certainement insuffisante ? L'étude de ces moments d'entre-soi féminin doit conduire à aller au-delà de ce qu'ils donnent à voir. Le sabar, dans le cadre de ces réunions féminines, semble d'abord être une leçon de séduction, une recette pour attraper les hommes que les femmes exercent, apprennent et enseignent, plus qu'une manière d'affirmer une résistance. Ces temps sont aussi l'occasion de parler des maris, des amants, des difficultés de la vie, sans pour autant qu'il y ait une mise en cause de l'ordre établi. Sans négliger le pouvoir de la danse, comme espace de défoulement, de démonstrations affectives et de décharge émotive, une autre proposition d'analyse peut être développée. Tout d'abord, l'effectivité de la domination masculine dans les sociétés ouest-africaines, exacerbée par l'islamisation, doit être relativisée. Si les hommes sont considérés comme des aînés et respectés en tant que tels, il faut distinguer le discours officiel, repris par tous et toutes, de la réalité de la pratique, car les femmes n'en gardent pas moins une grande liberté d'action, notamment sur la place publique du fait en particulier d'une relative indépendance économique (Le Carme 2000). En ce sens, les tours de danse féminins pourraient tout aussi bien être perçus comme les moments privilégiés d'une initiation diffuse à la féminité, qui transforme les femmes jusque dans leur corps, vecteur d'intériorisation des symboles, et les rend aptes à devenir de « véritables femmes », selon les normes culturelles en vigueur.

Les rites d'initiation, très souvent étudiés dans la littérature anthropologique africaniste, doivent régulièrement répondre à la nécessité de modifier en profondeur l'être des personnes destinées à accéder au statut d'adulte et à remplir les fonctions qui y sont liées :

De simples humains demeurés jusque-là dans l'état où, dirons-nous, la nature les a faits, sont transformés en des êtres dotés de propriétés « extraordinaires » qui les rendent aptes soit à devenir 'les véritables hommes' ou 'les véritables femmes' que les normes culturelles en vigueur ont définis. (Adler 2007 : 77)

Sous l'effet de l'histoire, la société wolof a abandonné les grands rites d'initiation collectifs masculins. Il n'existe pas d'équivalent féminin à cette institution. Les rites d'initiation féminins s'imbriquent dans ceux du mariage, notamment lorsque la jeune mariée rejoint le domicile conjugal. Si l'initiation féminine ne fait pas l'objet d'un marquage corporel comme la circoncision (opération au cœur du rituel de l'initiation masculine), il existait cependant des pratiques relatives au modelage du corps en ce qui concerne les jeunes filles devenues nubiles. Il s'agissait d'un rituel, dont l'opération principale consistait en un tatouage des gencives et des lèvres « njamm ». Au cours de ce rite de passage, effectué par une lawbe, la jeune fille était supposée maîtriser la douleur et montrer son courage par la danse. Pendant l'opération, les amies de la tatouée, appartenant à la même classe d'âge, l'encourageaient par des chants (Cissé 2006). Si aujourd'hui ce rituel spécifique a disparu, il ne reste pas moins diverses occasions de construire socialement les rôles et obligations féminins normés, dans une société qui reste marquée par la séparation des genres. Le marquage corporel du tatouage n'est plus, mais le corps continue d'être modelé, notamment par la danse du sabar qui initie les corps féminins à la sexualité comme une trace invisible mais indélébile. Par 
l'intermédiaire de la danse et des chants qui l'accompagnent, les jeunes filles touchent aux aspects de la féminité qui leur sont encore en partie voilés ${ }^{41}$. Le sabar les projette dans l'avenir. À travers ce qui est considéré comme un jeu, les mouvements du sabar ajoutent la préparation des corps à celle des esprits : les filles stimulent des muscles peu utilisés dans la vie courante et, en dansant, préparent leurs corps à leur future vie sexuelle ${ }^{42}$. Le sabar permet aussi une intériorisation des représentations des genres et ce, dès la plus tendre enfance, afin de préparer chaque « enfant du lignage » (Rabain 1994 [1979]) à la question du mariage et de l'union sexuelle. En offrant une image caricaturale de cette union, réduite à ses signifiants les plus crus, les performances des danseuses ne viennent-elles pas, en même temps, rappeler l'arbitraire des conventions sociales qui se greffent sur un acte aussi trivial?

\section{Références citées}

Adler, Alfred, 2007. «Initiation, royauté et féminité en Afrique Noire. En deçà ou au-delà de la différence des sexes : logiques politiques ou logiques initiatiques? », L'Homme, 183, p. 77-116.

Beidelman, Thomas, 1968. « Some Nuer notions of nakedness, nudity and sexuality », Africa, 38 (2), p. 113-132.

Bourdieu, Pierre,

1972. Esquisse d'une théorie de la pratique. Genève : Droz.

1987. Choses dites. Paris : Editions de Minuit.

Cissé, Momar, 2006. Paroles chantées ou psalmodiées wolof, collecte, typologie et analyse des procédés argumentatifs de connivence associés aux fonctions discursives de satyre et d'éloge. Dakar, thèse de l’Université Cheikh Anta Diop.

Diop, Abdoulaye, 1981. La société wolof, tradition et changement. Paris : Karthala.

Diouf, Jean-Léopold, 2003. Dictionnaire wolof-français et français-wolof. Paris : Khartala.

Duerr, H. P. 1992. Der Mythos vom Zivilisationsprozess. III. Obszönität und Gewalt. Frankfurt am Main : Suhrkamp.

DuPIRE, Marguerite, 1976. «Chasse rituelle, divination et reconduction de l'ordre sociopolitique chez les Serer du Sine (Sénégal) », L’Homme, 16 (I), p. 5-32.

Echard, Nicole, Odile Journet, Suzanne Lallemand, 1981. « De la prohibition à la prescription : sens et non-sens de la virginité des filles (Afrique de l'Ouest) », La Première Fois. Paris : Ramsay, p. 337-395.

Evans-Pritchard, Edward Evan, 1971. La femme dans les sociétés primitives et autres essais d'Anthropologie sociale. Paris : PUF.

FALl, Sokhna, 1998. Séduire : cinq leçons sénégalaises. Paris : éditions alternatives.

Faure, Sylvia, 2000. Apprendre par corps. Socio-anthropologie des techniques de danse. Paris : La Dispute.

Fürniss, Susanne, 2005. «Femmes, maîtresses, mères. Chants et danses des jeunes filles baka », Cahiers de musiques traditionnelles, 18, numéro thématique «Entre Femmes », p. 217-237.

HeAth, Deborah, 1994. « The politics of appropriateness and appropriation : recontextualizing women's dance in urban Senegal », American Ethnologist, 21 (1), p. 88-103.

41. Pour Pierre Bourdieu, il y a une manière de comprendre avec le corps qui se situe en deçà de la conscience discursive : on connaît sans avoir les mots pour le dire (notamment Bourdieu 1987 : 203).

42. Susanne Fürniss parle de «la structuration » des futures femmes baka par le chant et la danse. Elle démontre que l'apprentissage de la féminité et les activités de musique et de danse sont indissociablement liées (2005 : 217). 


\section{Audrey Dessertine}

Houseman, Michael, 2008. "Introduction », Systèmes de pensée en Afrique noire, 18, numéro "Éprouver l'initiation », p. 7-40.

Journet, Odile, 2008. «L'initiation mise en dérision (Sénégal, Guinée-Bissau) », Systèmes de pensée en Afrique noire, 18, numéro « Éprouver l'initiation », p. 165-192.

Lallemand, Suzanne, 1985. L'apprentissage de la sexualité dans les contes d'Afrique de l'Ouest. Paris : l'Harmattan.

Le CARme, Mireille, 2000. Marchandes dakaroises entre maison et marché, approche anthropologique. Paris : L'Harmattan.

Le Cour Grandmaison, Collette,

1978. La natte et le manguier. Paris : Mercure de France.

1967. Femmes dakaroises en milieu urbain. Abidjan : Annales de l'université d'Abidjan.

LegendRe, Pierre, 1978 (2000 pour la préface). La passion d'être un autre, étude pour la danse. Paris : Seuil.

LuLLI, Francesca, 2003. «Stratégies d'insertion urbaine : Associationnisme et éthique au féminin à Dakar », in Adriana Piga (éds), Islam et villes en Afrique au sud du Sahara. Paris : Karthala, p. 345-355.

Mauss, Marcel,

1950. Sociologie et anthropologie. Paris : PUF.

1921. « L'expression obligatoire des sentiments (rituels oraux funéraires australiens), Journal de psychologie 18, p. 421-437.

Moya, Ismael, 2004. «Entre démesure et tempérance. Argent et don au féminin à Dakar », in Drach Marcel, L'argent. Croyance, mesure, et spéculation. Paris : La Découverte, p. 167-180.

NdiAYe Raphaël, 1986. La place de la femme dans les rites au Sénégal. Dakar : NEA.

Neveu-Kringelbach, Hélène, 2007. « 'Le poids du succès' : Construction du corps, danse et carrière à Dakar », Politique Africaine, 107, p. 81-101.

Rabain, Jacqueline, 1994 [1979]. L'enfant du lignage, du sevrage à la classe d'âge. Paris : Payot.

Semin, Jeanne, 2007. «L'argent, la famille, les amies. Ethnographie contemporaine des tontines africaines en contexte migratoire », Civilisations, 56 (1-2), p. 183-199

Sow, Fatou, 1976. Femmes, sociabilité et valeurs africaines : la téranga dans la société wolof. Dakar : IFAN. 